\title{
NON-INTRUSIVE FORENSIC ANALYSIS OF VISUAL SENSORS USING OUTPUT IMAGES
}

\author{
Ashwin Swaminathan, Min Wu and K. J. Ray Liu \\ Electrical and Computer Engineering Department, University of Maryland, College Park.
}

\begin{abstract}
This paper considers the problem of non-intrusive forensic analysis of the individual components in visual sensors and its implementation. As a new addition to the emerging area of forensic engineering, we present a framework for analyzing technologies employed inside digital cameras based on output images, and develop a set of forensic signal processing algorithms for visual sensors based on color array sensor and interpolation methods. We show through simulations that the proposed method is robust against compression and noise, and can help identify various processing components inside the camera. Such a non-intrusive forensic framework would provide useful evidence for analyzing technology infringement and evolution for visual sensors.
\end{abstract}

\section{INTRODUCTION}

In the past two decades, innovations in information technology have contributed to a tremendous amount of economic growth around the world. Intellectual property protection plays a crucial role in fostering innovation, as it has been known for "adding the fuel of interest to the fire of genius" since the time of Abraham Lincoln. A growing amount of signal processing research in the recent years has been devoted to the security and protection of multimedia information. In contrast, the infringement problem of signal processing devices has received considerably less attention from the research community, yet fierce competition in the IT industry has led to an increasing number of infringement cases filed in U.S. courts. The remunerations awarded to successful prosecution have also grown tremendously, sometimes in billions of dollars [1].

According to the U.S. patent law, infringement of a patent consists of the unauthorized making, using, offering for sale or selling any patented invention. Patent infringement is usually difficult to detect, and even harder to prove in the court of law. The burden of proof often lies on patent holders, who are expected to provide solid evidence to substantiate their accusations. One common way to perform infringement analysis is to examine the design and implementation of a product and look for similarities with what have been claimed in existing patents, through some type of reverse engineering. However, this approach could be very cumbersome and ineffective, for example, it may involve going over VHDL design codes of an IC chip in charge of core information processing tasks, which is a daunting task even to the most experienced expert in the

Email contact: $\{$ ashwins, minwu, kjrliu\} @eng.umd.edu. field. Such analysis is often limited to the implementation of the idea rather than the idea itself, and thus could potentially lead to misleading conclusions [2].

Methods to identify patent infringements of signal processing device (such as camera and visual display) can be broadly classified into three main categories, based on the kind of input provided by the parties involved in dispute:

1. Intrusive Forensic Analysis: an analyst is able to access the device in question, disassemble it and carefully examine every part, including analyzing any available intermediate signals and states.

2. Semi-intrusive Forensic Analysis: an analyst has access to the device as a black box. He/she can design appropriate inputs to be fed into the device so as to gather forensic evidence about the working of its individual components.

3. Completely Non-Intrusive Forensic Analysis: An analyst is provided with only some sample data produced by the device, and analyzes them to gather evidence.

In this paper, we shall focus on visual sensors, for which we have witnessed a tremendous amount of development in the form of consumer-level digital camera. Intense competition on digital camera R\&D has been accompanied by numerous patent infringement cases related to digital imaging and visual processing employed by cameras. For example, Ampex Corporation has received more than $\$ 275$-million compensation from lawsuits and settlements involving patent infringement cases with many digital camera vendors [3].

As a new addition to the emerging field of forensic engineering, we present in this paper a framework for analyzing technologies employed inside a digital camera based on output images, and develop a set of forensic signal processing algorithms for visual sensors. Our algorithms are non-intrusive in nature, using only images captured by a given camera to estimate the type of techniques and parameters in various signal processing modules during the image acquisition process, and quantitatively determine the similarity and differences between cameras. For cameras from different vendors, the digital forensic knowledge obtained from such analysis can provide clues and evidence on technology licensing or infringement, which we shall refer to as infringement/licensing forensics. This will assist the enforcement of patent protection, which in turn would foster innovations in a long run. For cameras of the same brand but different models released at different years and/or at different price tiers, our analysis forms a basis of evolutionary forensics, as it can provide clues on the 
technology evolution that is valuable to the research and education in the technical community.

To our best knowledge, this is the first work introducing the concept of infringement and evolutionary forensics for camera solely through output images. Related prior art on non-intrusive image forensics falls in two main categories. In the forgery detection literature, there are recent works targeted at finding the type of post processing operations that occurs after an image has been captured by a camera, such as JPEG compression, Gamma correction, and copy-paste [4]. These methods do not concern the image creation process inside cameras. The problem of camera identification was studied very recently where noise patterns inherent to an image sensor were used as a unique identifier to each camera [5]. While useful in some forensic tasks, this approach is not for identifying common features tied to the same camera models, and thus cannot be directly applied to infringement and evolutionary study. Another blind-source approach employs statistics generated from similar images taken with different cameras to train classifiers for identifying the origin [6]. Although good results were reported in distinguishing pictures taken by three cameras, its ability to differentiate a large number of cameras under diverse training sets is unclear. As shall be seen from our results later in the paper, by striving to acquire knowledge on the inner working of each camera from output images, our proposed forensic framework can also support more accurate identification for a large number of cameras.

The paper is organized as follows. After reviewing a model of cameras' scene capture process in Section 2, we focus on color sensor pattern and interpolation aspects, and present new algorithms to estimate them from output images. Section 3 first shows the experimental results from synthetic data to validate the proposed algorithm, and then presents forensic analysis results on data set from 16 cameras.

\section{MODELLING AND PARAMETER ESTIMATION}

Fig. 1(a) shows the image capture process. The rays from the scene pass through the lens and the optical filters and are finally recorded by the charge coupled device (CCD) detectors. Most digital cameras use a color filter array (CFA) to sample the real-world scene. Some examples of CFA patterns are shown in Fig. 1(b). The CFA consists of an array of color sensors, each of which captures the corresponding color of the real-world scene at an appropriate pixel location where it is located. The remaining pixel values are interpolated using the sampled data. CFA interpolation (or demosaiking) is an important step to maintain the quality of the final output image $[8,9]$. After interpolation, the three images corresponding to the red, green and the blue components go though a postprocessing stage. In this stage, white balancing and color correction are done to remove unrealistic color casts so that objects that appear white in reality appear white in the photograph. Finally, the image may be JPEG compressed to reduce storage space.

Our present work focuses on CFA and the associated interpolation. Based on minimum mean-square estimate, we identify the CFA pattern and the type of interpolation, as well as estimate the interpolation parameters. A random CFA pattern - $p$ is initially assumed. Using $p$ and the final camera output $S_{d}$, we obtain an estimate for $S_{d}$ and find the interpolation error. The pattern that gives the lowest error is declared to be the correct CFA pattern.

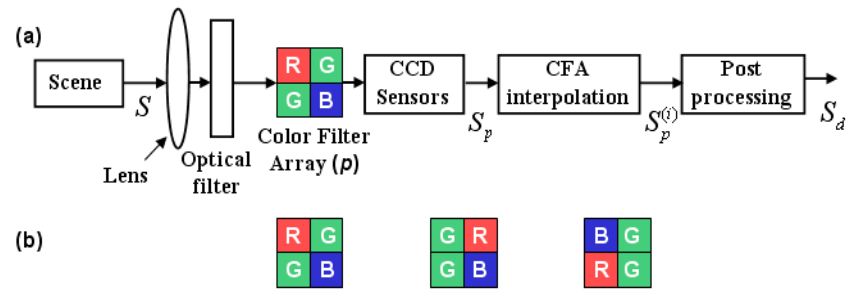

Fig. 1. (a) Scene Capture Process in a Camera; (b) Sample Color Filter Arrays.

Estimating Interpolation Coefficients In the absence of post-processing, the output digital image $S_{d}$ provides partial information about the sampled real-world picture $S_{p}$. Furthermore, from our preliminary study, we observe that most of the interpolation algorithms can be well approximated to be close to linear in specific regions of the image. Hence, we divide the picture based on the gradient values in a local neighborhood and obtain the interpolation coefficients in each region using a linear model. Let us define $I_{i, j}=S_{d}(i, j, p(i, j))$. The horizontal and vertical gradients at the location $(i, j)$ can be found using

$$
\begin{aligned}
H_{i, j} & =\left|I_{i, j-2}+I_{i, j+2}-2 I_{i, j}\right|, \\
V_{i, j} & =\left|I_{i-2, j}+I_{i+2, j}-2 I_{i, j}\right| .
\end{aligned}
$$

The image pixel at location $(i, j)$ is grouped together into one of the three categories based on the gradient values in its neighborhood: Region 1 contains pixels with a significant horizontal gradient for which $\left(H_{i, j}-V_{i, j}\right)>T$ where $T$ is a suitably chosen threshold; Region 2 contains the set of pixels with a significant vertical edge $\left(V_{i, j}-H_{i, j}\right)>T$; and Region 3 includes the remaining parts of the image and primarily contains smooth regions.

Using the final camera output $S_{d}$, we obtain a set of linear equations for all the pixels in each region separately by representing the interpolated pixels as a linear combination of its surrounding pixel values. Let these equations be represented in the form $\mathbf{A x}=\mathbf{b}$. JPEG compression and other post-processing operations would bring distortions to the values of $\mathbf{A}$ and $\mathbf{b}$ and thus affect the estimate of the interpolation coefficients. Hence in order to make the solution robust to noise, we formulate and solve the following problem:

$$
\min _{\mathbf{E}, \mathbf{r}}\left\|\left[\begin{array}{ll}
\mathbf{E} & \mathbf{r}
\end{array}\right]\right\|_{F} \quad \text { subject to }[\mathbf{A}+\mathbf{E} \mathbf{b}+\mathbf{r}]\left[\begin{array}{c}
\mathbf{x} \\
-1
\end{array}\right]=0
$$


Here $\|.\|_{F}$ denotes the Frobenius norm of the matrix. The solution to the minimization problem can be written as

$$
\left[\begin{array}{c}
\mathbf{x} \\
-1
\end{array}\right]=-\frac{1}{v_{n+1, n+1}} \mathbf{v}_{n+1}
$$

where $\mathbf{v}_{n+1}$ represents the $(n+1)^{\text {th }}$ right singular vector of the combined matrix $\left[\begin{array}{ll}\mathbf{A} & \mathbf{b}\end{array}\right]=\mathbf{U} \Sigma \mathbf{V}^{T}$.

Estimating CFA pattern Once the interpolation coefficients are obtained, it can be used to re-interpolate the sampled CFA output $S_{p}$ in the corresponding regions to obtain an estimate of the final output image $\hat{S}_{d}^{(p)}$. The difference between $\hat{S}_{d}^{(p)}$ and the actual camera output $S_{d}$ gives the interpolation error. This error is obtained for all candidate search patterns and the pattern $\hat{p}$ that gives the lowest overall absolute value of error is chosen as the estimated CFA.

The choice of the candidate search patterns can be done based on prior knowledge on the possible patterns used in cameras. We note that most commercial cameras use the RGB type of CFA with a fixed periodicity of 2 . If we confine our search to these CFA patterns, then we will require a total of $3^{4}$ candidate patterns. Further, we show that the number of searches required can be reduced to around 15 using a tree based search.

\section{FORENSIC ANALYSIS AND RESULTS}

\subsection{Forensic Analysis on Synthetic Data}

As an initial step, we use synthetic data to study the performance of the proposed techniques and to demonstrate the proof of concept. We use around 20 representative images. The original images were first down-scaled to remove the effect of previously applied filtering and interpolation operations and then sampled on the three different CFA patterns as shown in Fig. 1(b). Each of the sampled images were then interpolated 6 different interpolation algorithms: (1) Bilinear, (2) Bicubic, (3) Smooth Hue, (4) Median Filter, (5) Gradient based, and (6) Adaptive Color Plane [8, 9]. Thus, our total dataset contains 360 images each of size $512 \times 512$.

In the noiseless case under no post-processing, we observed no errors in estimating the CFA pattern. The interpolation coefficients corresponding to the three color planes (and the three regions) were estimated and a support vector machine (SVM) was used for identifying the interpolation method. Randomly chosen 8 images under all interpolation techniques was used as ground truth in training and testing was done on the remaining 12 pictures. We obtained $100 \%$ classification accuracy in identifying the correct CFA interpolation algorithm with no false alarms.

Among various post-processing inside camera, multiplicative operations such as white-balancing and color correction do not affect the solutions to the linear equations for interpolation estimation as the same factor is applied to both sides

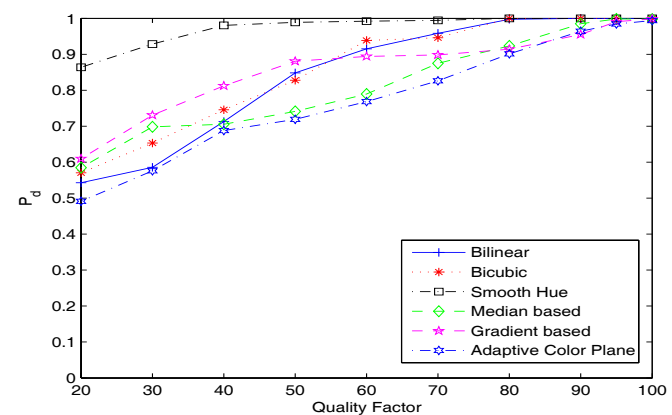

Fig. 2. Probability of correctly identifying the CFA interpolation technique for different JPEG quality factors under Multiple Camera Multiple Scene (MCMS).

of the equation. As such, we focus on JPEG compression (quality factors 20-100) and additive noise (PSNR 15-40dB). We observed that in all cases, our proposed MMSE estimator gave very good results and the correct CFA pattern was identified in most cases. On the whole, we obtained only one error in estimation - when the PSNR was $15 \mathrm{~dB}$ and the adaptive color plane interpolation method was used. However, in this case, it was found that the correct pattern came in the top three choices. Next, we study the performance of the CFA interpolation identification algorithm when the final camera output images are JPEG compressed. Two scenarios are considered: (1) Multiple Camera Multiple Scene (MCMS), where pictures obtained from different cameras come from different scenes; this corresponds to the completely non-intrusive scenario discussed in Section 1; (2) Multiple Camera Single Scene (MCSS), where a forensic analyst has access to each camera and is able to use the different cameras to capture similar scenes; this corresponds to the semi-intrusive forensic analysis discussed in Section 1.

The performance of the proposed CFA interpolation technique for the MCMS for different JPEG quality factors is shown in Fig. 2. Here we use 12 images each under a randomly chosen interpolation technique for training ( 2 for each interpolation method) and the remaining 48 images for testing. We observe that the probability of correctly estimating the interpolation technique used is around $85 \%$. We have also tested the multiple camera single scene case, where the probability of correctly estimating the interpolation technique is found to have improved to around $96 \%$, and thus the forensic analyst can make the decision with a higher confidence level.

\subsection{Forensic Analysis on Camera Data Set}

Our study has considered 16 different cameras models as shown in Table 1. Around 40 different images were taken from each camera. The images were taken under completely uncontrolled conditions, i.e. different sceneries, different lighting, and compressed under different quality levels as built in each camera.

16-Camera Classifier We first estimate the CFA pattern from the output images using the proposed algorithm. We 
Table 1. Camera Models used in Simulation

\begin{tabular}{c|l||c|l}
\hline No. & Camera Model & No. & Camera Model \\
\hline 1 & Canon A75 & 9 & Sony Cybershot \\
2 & Canon Powershot S400 & 10 & Sony P72 \\
3 & Canon Powershot S410 & 11 & Olympus C3100Z \\
4 & Canon Powershot S1 IS & 12 & Olympus C765UZ \\
5 & Canon Powershot G6 & 13 & Minolta DiMage S304 \\
6 & Canon EOS Dig. Rebel & 14 & Casio QV-UX2000 \\
7 & Nikon E4300 & 15 & FujiFilm S3000 \\
8 & Nikon E5400 & 16 & Epson PhotoPC 650 \\
\hline
\end{tabular}

observe that all the cameras use the Bayer color filter array (shown in the left corner in Fig. 1(b)). This is the most popular CFA pattern. Here, the red and the blue colors are sampled on a rectangular grid and the green color corresponding to the luminance component of the image is more finely sampled on a hexagonal grid to reduce the effects of aliasing [7].

We then estimate the CFA interpolation coefficients using the proposed algorithm. We use a classification based model to study the closeness among the set of coefficients. If two sets of coefficients are similar, then in most cases they should get classified into similar classes while testing. The simulation results with an SVM based classifier with 25 training images are shown in Fig. 3 in terms of a confusion matrix. The $(i, j)^{t h}$ element in the confusion matrix gives the probability of being classified as camera $-j$ when the picture actually comes from camera- $i$. The table shows only statistically significant values that are greater than $\lambda=\frac{1}{N_{c}}$, where $N_{c}$ is the number of cameras. The 16-camera classification results indicate that we can distinguish cameras based on the estimated interpolation coefficients and therefore it is highly likely that cameras from different companies differ in the detailed ways of interpolation especially when handling edge regions.

We also notice a great degree of similarity among the cameras from the same company (e.g. Canon models 1-6) indicating that they are probably using similar kinds of interpolation methods. To explore further, we grouped all images from the same company into one class and built a 8-class classifier. We observed that the overall classification accuracy improved to $95 \%$, which validates our conjecture.

Similarity Between Different Camera Models We start with cameras from the same vendor, and conduct a case study on two successive releases of Canon 4M-pixel models, S400 and S410. We train a SVM classifier using the images obtained from 15 cameras except Canon S410 and then test it using the images from Canon S410. The results show that $72 \%$ of the Canon S410 images are classified as Canon S400 and an additional $25 \%$ of the $\mathrm{S} 410$ images are classified as one of the other Canon models. This suggests that interpolation algorithms used by Canon S400 and S410 have a high degree of similarity. We conducted similar tests for cameras from different vendors. We observe that around $78 \%$ of the Minolta DiMage S304 pictures are classified as Nikon E4300 camera model when Minolta model was not used in training. This suggests that the interpolation parameters of the two cameras

\begin{tabular}{|c|c|c|c|c|c|c|c|c|c|c|c|c|c|c|c|c|}
\hline & 01 & 02 & 03 & 04 & 05 & 06 & 07 & 08 & 09 & 10 & 11 & 12 & 13 & 14 & 15 & 16 \\
\hline 01 & 0.89 & $*$ & $*$ & $*$ & $*$ & $*$ & $*$ & $*$ & $\pi$ & $*$ & $*$ & $*$ & $*$ & $*$ & 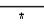 & $*$ \\
\hline 02 & * & 0.61 & 0.18 & * & 0.08 & * & * & * & * & * & * & * & * & * & " & $\pi$ \\
\hline 03 & * & 0.20 & 0.70 & \# & * & \# & \# & * & \# & \# & * & \# & \# & \# & * & \# \\
\hline 04 & * & 0.10 & ${ }^{*}$ & 0.73 & 0,07 & \# & * & * & \# & \# & 末 & \# & \# & * & * & \# \\
\hline 0.5 & \# & 0.12 & 0.16 & \# & 0.56 & 0.08 & \# & 末 & \# & \# & \# & \# & \# & \# & * & \# \\
\hline 06 & * & * & $\star$ & 0.08 & ${ }^{\star}$ & 0.83 & $\star$ & $\star$ & 末 & * & * & * & 末 & * & * & $\star$ \\
\hline 07 & * & $t$ & * & $*$ & $\hbar$ & * & 0.81 & * & * & 0.07 & * & * & \# & * & * & * \\
\hline 08 & * & * & * & * & * & * & $*$ & 0.89 & $*$ & $*$ & * & * & * & * & , & $*$ \\
\hline 09 & * & $*$ & * & $\pi$ & * & $\pi$ & $\pi$ & $\pi$ & 0.93 & $\pi$ & * & * & * & * & * & $\pi$ \\
\hline 10 & \# & $\hbar$ & \# & \# & \# & \# & \# & \# & \# & 0.82 & $\hbar$ & \# & $\hbar$ & $\hbar$ & \# & $\hbar$ \\
\hline 11 & * & t & * & * & * & * & \# & * & t & 0.09 & 0.88 & $\hbar$ & * & * & * & * \\
\hline 12 & * & * & \# & \# & \# & \# & \# & * & 末 & * & $\star$ & 0.88 & * & * & * & \# \\
\hline 13 & $\star$ & t & * & * & * & $\star$ & 0.07 & * & * & * & $\hbar$ & ${ }^{*}$ & 0.89 & * & * & $\hbar$ \\
\hline 14 & * & * & * & $\star$ & \# & * & * & * & 末 & * & * & * & * & 0.93 & * & * \\
\hline & * & * & * & * & * & * & * & * & * & * & * & * & * & * & 0.94 & * \\
\hline & $\pi$ & $\pi$ & * & * & * & $\pi$ & * & * & * & $\pi$ & * & $\pi$ & * & * & " & 1.00 \\
\hline
\end{tabular}

Fig. 3. Confusion Matrix for all cameras numbered as in Table 1 . The matrix is divided based on different camera makes. The values below $\lambda=\frac{1}{16}$ are denoted by $*$.

are likely to be similar.

\section{SUMMARY AND CONCLUSIONS}

In this paper, we consider the problem of infringement forensics of visual sensors. We develop methods to find the different building blocks of the camera based on sample camera output images and detailed studies of the scene capture process. We focus on techniques for identifying the CFA pattern and the interpolation algorithm. We show through detailed simulations that the proposed methods are robust to various kinds of post-processing that might occur in the camera and are thus applicable in real world situations. These techniques are then used to gather forensic evidence on real world datasets captured by cameras of 16 different models under various situations. We believe that such forensic study would be great interest for analyzing technology infringement and evolution for visual sensors.

\section{REFERENCES}

[1] A. B. Jaffe, and J. Lerner, "Patent Prescription: A radical cure for the ailing U.S. patent system," News article available online at http: //www. spectrum. ieee.org/careers /

[2] D. F. McGahn, "Copyright Infringement of Protected Computer Software: An Analytical Method to Determine Substantial Similarity," Rutgers Comp. \& Tech. Law Jrnl., Vol. 21, No. 1, pp. 88-142, 1995.

[3] "How Ampex Squeezes Out Cash: It's suing high-tech giants that rely on its patents," Online news, http://www.businessweek.com/, April 2005.

[4] A. C. Popescu and H. Farid, "Statistical Tools for Digital Forensics," Proc. of the $6^{\text {th }}$ Info. Hiding Workshop, Toronto, Canada, 2004.

[5] J. Lukas, J. Fridrich and M. Goljan "Determining Digital Image Origin Using Sensor Imperfections," Proc. of the SPIE, pp. 249-260, Jan 2005.

[6] M. Kharrazi, H. T. Sencar, N. Memon and I. Avcibas, "Blind Camera Identification Based on CFA Interpolation," Proc. IEEE Intl. Conference on Image Processing (ICIP), 2005.

[7] J. Adams, K. Parulski, K. Spaulding, "Color Processing in Digital Cameras,” Proc. of IEEE Micro, Vol. 18, No. 6, pp. 20-30, Nov 1998.

[8] J. Adams, "Interaction between Color Plane Interpolation and other Image Processing Functions in Electronic Photography," SPIE Cameras and Sys. for Electronic Photography \& Scientific Imaging, Feb 1995.

[9] A. C. Popescu and H. Farid, "Exposing Digital Forgeries in Color Filter Array Interpolated Images," IEEE Trans. on Signal Processing, Vol. 53, No. 10, pp. 3948 - 3959, Oct 2005. 